\title{
Analyse De La Variation Morpho-Phenologique Et Genetique De Vingt Accessions Du Ble Dur Algerien (Triticum durum D.E.S.F.)
}

\author{
Rima Belattar, M.A.A \\ Département de Biologie et Ecologie Végétale, Faculté des Sciences de la \\ Nature et de la Vie, Université Ferhat Abbas Setif, Algérie \\ Leila Boudour, Prof. \\ Ghania Chaib, PhD \\ Département de Biologie et Ecologie Végétale, Faculté des Sciences de la \\ Nature et de la Vie, Université Frères Mentouri Constantine 1 Algérie
}

doi: 10.19044/esj.2016.v12n24p168 URL:http://dx.doi.org/10.19044/esj.2016.v12n24p168

\begin{abstract}
Durum wheat (Triticum durum Desf.) is a strategic culture in Algeria. Characterization and evaluation of crop varieties allow backup and restoration of this genetic heritage and its use in breeding programs.

In this context, the study is subject to the identification of some morpho phenological parameters and molecular markers in kind RAPD variability of 20 genotypes of collection of Algerian durum wheat (Triticum durum Desf.) Belong to two varieties (rechenbachi and leucomelan). The results of the morpho-phenological and genetic analysis show substantial phenotypic diversity between varieties. RAPD marker showed an intervarietal genetic polymorphism.
\end{abstract}

Keywords: durum wheat (Triticum durum), phenological, morphological, RAPD markers, genetic polymorphism.

\section{Resumé}

Le blé dur (Triticum durum Desf.) est une culture stratégique en Algérie. La caractérisation et l'évaluation des variétés cultivées permettent la sauvegarde et la réhabilitation de ce patrimoine génétique ainsi que son utilisation dans des programmes de sélection. Dans ce contexte, l'étude a fait objet de la mise en évidence de quelques paramètres morpho- phénologiques, et des marqueurs moléculaires type RAPD dans la variabilité d'une collection de 20 génotypes de blé dur algérien (Triticum durum Desf.) appartiennent à deux variétes (rechenbachi et leucomelan).Les résultats de l'analyse morpho-phénologique et génétique montrent une importante 
diversité phénotypique entre les variétés. Les marqueur RAPD ont mis en évidence un polymorphisme génétique intervariétale.

Mots clés : blé dur (Triticum durum), phénologiques, morphologiques, marqueurs RADP, Polymorphisme génétique.

\section{Introduction}

Triticum turgidum L. subsp. Durum (Desf.) est l'une des céréales les plus anciennes et les plus cultivées dans le monde avec plus de 17 millions d’hectares. Cette éspece présente un grand marché d’importation. De ce fait, elle est considerée comme une culture de base pour le bassin méditerranéen. Ceci est dû à la grande consommation des méditerranéens de dérivés de blé dur et son adaptation à des environnements semi-arides (Nazco et al., 2012).

L'amélioration d'une espèce cultivée repose essentiellement sur la gestion et l'exploitation de la variabilité agromorphologique et génétique qu'elle renferme. L’éventail de la variabilité génétique des blés cultivés, résultat de 10000 an de culture, a considérablement diminué ces dernières années en raison de l'introduction des techniques scientifiques modernes pour les besoins de la sélection.

L’amélioration génétique des plantes pour une meilleure adaptation aux contraintes environnementales reste prometteuse. En Algérie, la sélection du blé dur s’est faite fortement dans les introductions des centres internationaux de la recherche agronomique. Les variétés sélectionnées réussissent bien dans les plaines intérieures et sur le littoral. Cependant le niveau de rendements en grains reste très variable sur les hautes plaines (Bouzerzour et al., 2002) contrairement aux marqueurs traditionnels: morphologiques et biochimiques. Ici, les marqueurs moléculaires ne sont pas influencés par les fluctuations de l'environnement et sont indépendants de l'organe analysé et du stade de développement de la plante. Ces marqueurs moléculaires deviennent aujourd'hui un outil essentiel de l'amélioration des plantes et ouvrent de nouvelles perspectives pour le sélectionneur. (Hospital, 2001 ; Moreau et al., 2001 ; Eagles et al. , 2001 ; Dekkers et Hospital, 2002).

L'utilisation des marqueurs RAPD pour l'identification des cultivars à travers le profilage ADN est utile pour mesurer la variation génétique dans les collections de matériel génétique. Les marqueurs RAPD sont des marqueurs dominants et sont largement utilisés dans la cartographie génétique et l'identification des locis liés aux différents traits (Sun et al .2003).

En raison de sa simplicité technique et de sa vitesse de réalisation, la méthodologie RAPD est largement utilisée pour l'analyse de la diversité et l'identification du génotype dans différentes plantes de culture. 
Notre travail s'inscrit dans le cadre d'une étude de la valorisation des ressources phytogénétiques des blés par l'analyse de la variation morphophénologique et le polymorphisme génétique d'un ensemble des génotypes appartenant à deux variétés de blé dur (reichenbachi et leucomelan).

\section{Materiel et Méthodes Matériel végétal}

Le matériel d'étude est constitué de 20 accessions de blé dur algérien appartenant aux variétés reichenbachi (R87, R126, R35, R114, R77, R34, R4, R63, R75, R89) et leucomelan (L192, L111, L172, L138, L195, L201, L139, L174, L150, L125). Les principaux caractéristiques des deux variétés étudiées sont présentées au tableau $n^{\circ} 1$ (Boudour, 2004 ; 2006).

Tableau nº ${ }^{\circ}$ : Caractéristiques des variétés étudiées (Boudour, 2004 ; 2006).

\begin{tabular}{|l|l|}
\hline Variétés & \multicolumn{1}{c|}{ Caractéristiques } \\
\hline leucomelan & $\begin{array}{l}\text { Epi blanc, glabre ou lisse, de forme pyramidale, triangulaire aplatie, } \\
\text { parfois carré ou rectangulaire avec un noircissant sur le dos des glumes } \\
\text { glabres, d'une compacité assez faible parfois plus marquée. Les glumes } \\
\text { sont prolongées par des barbes noires. Le grain est blanc gros, allongé et } \\
\text { bossu. La paille est généralement creuse ou demi-creuse à 3/4 creuse. }\end{array}$ \\
\hline reichenbachi & $\begin{array}{l}\text { Epi blanc glabre, assez lâche, droit,étroit, à barbes noires faiblement } \\
\text { divergentes, à grain rouge, de taille moyenne et bossu et à paille creuse } \\
\text { ou demi-creuse à 3/4 creuse. }\end{array}$ \\
\hline
\end{tabular}

\section{Mise en place de l'expérimentation}

L'expérimentation est réalisée sur une parcelle relativement homogène, au niveau de la station expérimentale (ITGC) d'Elkhroub, située au sud de Constantine, Algérie, à une altitude de $640 \mathrm{~m}$. Le sol est profond et caillouteux avec une texture argilo-limoneuse, $\mathrm{P}^{\mathrm{H}}=7,5$.

Le semis est fait manuellement à une profondeur de 3 à $5 \mathrm{~cm}$. Chaque variété est séparée de l'autre par deux lignes de seigle et chaque génotype contient trois lignes (répétitions).

La pluviométrie de la campagne était dans l'ensemble de 519,5 mm entre septembre et mai. Les températures moyennes mensuelles sont normales variant respectivement de $6,77 \mathrm{C}^{\circ}$ à $7,29 \mathrm{C}^{\circ}$ en hiver et de 17,54 $\mathrm{C}^{\circ}$ à $22,53 \mathrm{C}^{\circ}$ en été. Elles ont été un peu fraiches durant l'hiver sans avoir d'effets nefastes sur les plantes.

\section{Paramètres mesurés}

L'étude a porté sur trois axes des paramètres:

Paramétres phénologiques

Les parametres phénologiques sont présentés par la durée d'épiaison, qui est notée lorsque 50\% d'éléments étudiés (talle, épi...) de la parcelle élémentaire sont sorties. 
Paramétres morphologiques

Les parametres morphologiques sont mesurés durant le cycle de développement de la plante à partir du semis jusqu'à maturité. Ces paramètres sont: La hauteur de la plante (HP mesuré du sol jusqu'au sommet de l’épi (barbe non incluse), La longueur de l’épi (LE) à la maturité, mesuré de la base de l'épi jusqu'au sommet, barbe non incluse, La longueur de la barbe (LB) mesurée à maturité à partir du tiers moyen de l'épi et La longueur du col (LC) mesurée à la maturité à partir du dernier nœud.

Paramétres génétiques

L'étude moléculaire consiste en utilisation de la technique RAPDPCR. Ce travail est réalisé au laboratoire de protection et d'amélioration des plantes au centre de biotechnologie de Sfax, Tunisie. L'extraction d'ADN est faite suivant la méthode de Dellaporta et al., (1983) à partir de feuilles issues de chaque variété broyées en présence de l'azote liquide. Les broyats sont mis dans des tubes Eppendorf 2ml, additionnés $1 \mathrm{ml}$ de tampon d'extraction Tris-HCl, (100 mM Tris-HCl (pH8), 500 mM NaCl, 50 mM EDTA, 1,25\% SDS). Mélanger, et mettre au bain marie à $65^{\circ} \mathrm{C}$ pendant $1 \mathrm{~h}$. Centrifugation à $12000 \mathrm{tr} / \mathrm{min}$ à $4^{\circ} \mathrm{C}$ pendant $15 \mathrm{~min}$. Additionner $1 \mathrm{ml}$ chlorophorme $\mathrm{A} / \mathrm{A}$ (24/1), laisser en agitation dans la roue pendant $15 \mathrm{mn}$. Après centrifugation à $3000 \mathrm{tr} / \mathrm{min}$ à $4^{\circ} \mathrm{C}$ pendant $15 \mathrm{~min}$, le surnageant est transféré à un nouveau tube eppendorf $1,5 \mathrm{ml}$. Additionner $1 \mathrm{ml}$ éthanol absolu froid, mélanger, mettre au congélateur à $-20^{\circ} \mathrm{C}$ pendant 30 à $20 \mathrm{mn}$ pour la précipitation. Après récupérer l'ADN dans des nouveaux tubes avec cône jaune stérile, le surnageant est éliminé et le culot lavé deux fois avec $500 \mu$ l d'éthanol $70 \%$. Puis centrifuger à $5000 \mathrm{tr} / \mathrm{min}$ à $4^{\circ} \mathrm{C}$ pendant $5 \mathrm{~min}$, le surnageant est éliminé et le culot laissant séché pendant $30 \mathrm{~min}$, puis additionner $100 \mu \mathrm{l} \mathrm{H}_{2} \mathrm{O}$ et laisser les tubes dans la chambre froide une nuit en agitation balançoire.

L'analyse des marqueurs RAPD est effectuée par l'utilisation de deux amorces aléatoires indiquées dans le tableau $n^{\circ} 2$.

Tableau $n^{\circ} 2$ : Les codes des amorces, leurs séquences et leurs pourcentage en GC.

\begin{tabular}{|c|c|c|}
\hline Nom d'amorce & Séquence & CG\% \\
\hline DuPw004 & 5'-GGTCTGGTCGGAGAAGAAGC-3' & $60 \%$ \\
\hline DuPw023 & 5'-TTGCTCCGATGTAATAAGCG-3 & $45 \%$ \\
\hline
\end{tabular}

La PCR est réalisée dans un volume final de $50 \mu$ l contient : $1 \mu l$ d' ADN, $5 \mu l$ de Tampon 10x, $2 \mu$ l Dntp, $2 \mu$ Amorce1 (10 $\mu \mathrm{M}), 2 \mu l$ Amorce 2

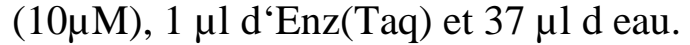

L'amplification de l'ADN à l'aide de la technique RAPD-PCR est réalisée selon la méthode de Williams et al., (1990) avec des modifications. Cette amplification comprend une phase de dénaturation initiale d'ADN de 2 min à $94^{\circ} \mathrm{C}$, une phase de 40 cycles avec 1 min à $94^{\circ} \mathrm{C}$ pour la dénaturation 
de l’ADN, 1 min à $55^{\circ} \mathrm{C}$ pour l'hybridation des amorces, 1 min à $72^{\circ} \mathrm{C}$ pour l'élongation et une phase d'élongation finale de $10 \mathrm{~min}$ à $72^{\circ} \mathrm{C}$.

\section{Analyse statistique}

L’étude phéno-morphologique est réalisée grace à une analyse de la variance (ANOVA un facteur et 2 facteurs) à $\mathbf{l}^{\text {' }}$ aide de logiciel XLSTAT version 10. L'étude moleculaire est analyseé par les Matrix de similarité et les Dendrogrammes avec le logiciel SPSS version 9.

\section{Resultats et Discussion}

\section{Paramètre phénologique : durée d'épiaison}

La durée d’épiaison est convergente chez toutes les accessions pour chaque variété et divergente entre les deux variétés. Elle varie de 140 jours chez le génotype G4 à 142 jours chez les autres génotypes de la variété rechenbachi. Alors qu'elle s'établi entre 135 jours chez le génotype G111 à 136 jours chez les autres génotypes de la variété leucomelan (Fig.1). La date d'épiaison, souvent utilisée comme un indicateur de précocité. Cette date est considérée comme un caractère important, qui a un effet intéressent sur les rendements des céréales, surtout en zone où la distribution de la pluviométrie et la variabilité des températures affectent la longueur du cycle de développement (Mekliche,1983 ;Hadjchristodoulou,1987).

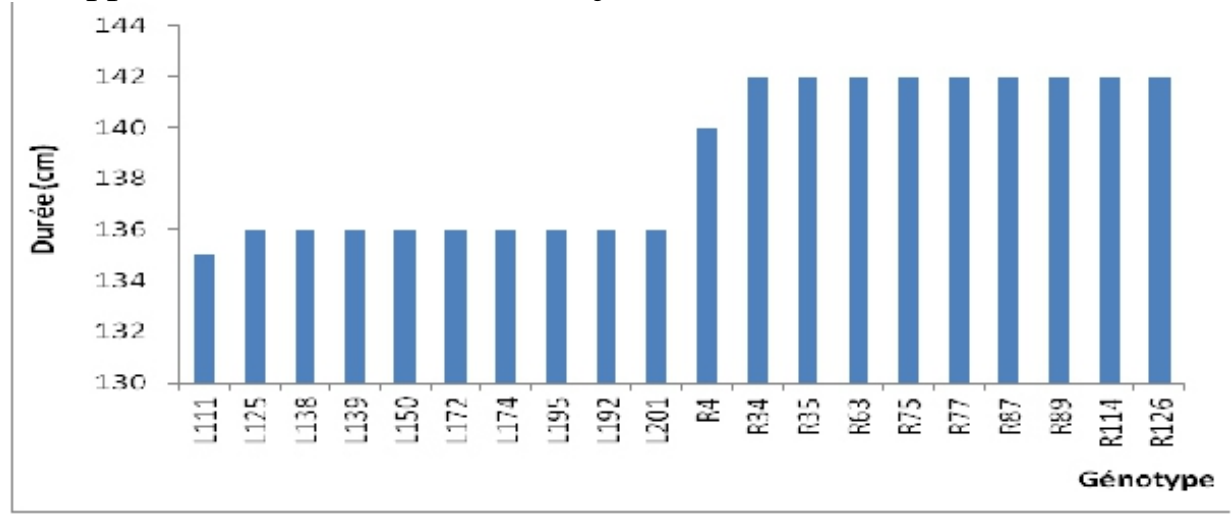

Fig. 1. Durée d’épiaison de vingt génotypes de blé dur

\section{Paramètres morphologiques}

L'étude des quatre paramètres morphologiques montre une correlation importante entre eux. Les génotypes les plus longs ayant respectivelent la longueur du col, la longueur d'épi et la longueur du barbes sont trés intéressents et en particulier le génotype L138 qui a une hauteur du plante avec une longueur du barbes importants, une longueur du col et une longueur d'épi faible en comparaison avec les autres génotypes (Fig.2). 


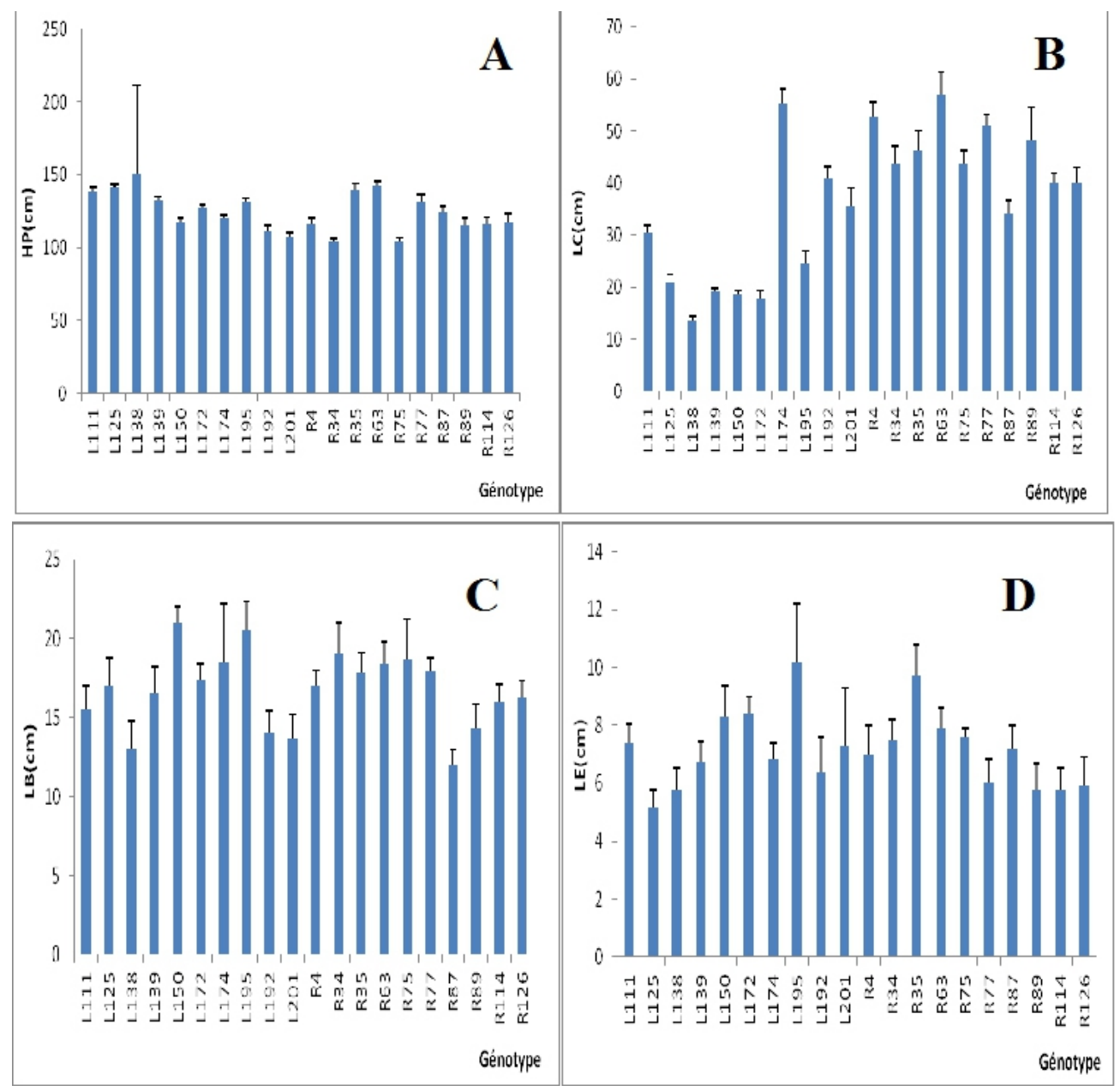

Fig.2. Paramètres morphologiques des vingt génotypes de blé dur :A: Hauteur de la plante, B: Longueur du col de l'épi , C: Longueur des barbes, D: longueur de l'épi

L'étude statistique des paramétres morphologiques étudiés révele une difference trés hautement significatives pour les trois paramétres (LE, LB et LC) et hautement significatif pour le paramétre HP (Tableau n³ ).

La hauteur des plantes varie de $104 \mathrm{~cm}$ à $151 \mathrm{~cm}$ chez les deux variétés leucomelon et rechenbachi (Fig. 2A). L'analyse de la variance à un facteur montre une différence significative et met en évidence trois groupes de génotypes :

Tableau $\mathrm{n}^{\circ} 3$ : Le test NWK des parametres morphologiques

\begin{tabular}{|c|c|c|c|c|c|}
\hline source & ddl & SCM & $\mathrm{CM}$ & F de Fisher & $\operatorname{Pr}>F$ \\
\hline HP & 19 & 10823,703 & 569,669 & 2,950 & $0,002 * *$ \\
\hline$\overline{\mathrm{LE}}$ & 19 & 95,877 & 5,046 & 4,961 & $<0,0001^{*} * * *$ \\
\hline LB & 19 & 335,213 & 17,643 & 6,304 & $<0,0001^{* * * *}$ \\
\hline $\mathrm{LC}$ & 19 & 10676,556 & 561,924 & 70,465 & $<0,0001^{* * * *}$ \\
\hline
\end{tabular}


Le premier groupe comprend le génotype L138 avec une hauteur de 151,33 cm. Le deuxième groupe composé des génotypes R63, L125, R35, L111, L139, L195, R77, L172, R87, L174, L150, R126, R114, R4 et R89 qui ont une hauteur des plantes importante allant de $110.80 \mathrm{~cm}$ à $142.60 \mathrm{~cm}$. Le troisième groupe forme les plantes les plus courtes L201, R75 et R34 avec une moyenne de $104 \mathrm{~cm}$ à 107,400 cm.

Nachit et al.(1986) in Bouzerzour et Oudina.(1989) ont montré que la hauteur est un caractère désirable en zone semi-aride où la verse n'est pas un problème. De plus, dans ces zones, la paille est très appréciable par le cheptel.

Le col de l’épi des deux génotypes R63 et L174 est plus élevé avec une moyenne qui varie de 56,9 cm à 55,2 cm suivie par les deux génotypes R4 et R77 avec une moyenne entre $52,7 \mathrm{~cm}$ et $52 \mathrm{~cm}$. Les trois groupes intermédiaires englobent les génotypes R89, R35 et R114 d'un intervalle variant de 48,4 cm à $44 \mathrm{~cm}$. Les deux derniers groupes se distinguent par les deux génotypes L172 et L 138 avec respectivement le plus bas col d’épi de $17,60 \mathrm{~cm}$ et $13,50 \mathrm{~cm}$.

La longueur d'épi se situe entre $5,5 \mathrm{~cm}$ et $10,2 \mathrm{~cm}$. La longueur moyenne est de 6,47 cm chez la variété leucomelon, alors qu'elle est de 7,04 $\mathrm{cm}$ chez la variété reichenbachi. L’analyse de la variance révèle cinq groupes distincts:

- Le premier groupe comprend le génotype L195, exprimant la valeur la plus élevée;

- Le deuxième, troisième et quatrième groupe dont les valeurs sont moyennement élevées variant de 7,2 à 9,7 cm.

Le cinquième groupe est constitué de 11 génotypes R4, L174, L139, L111, L192, R77, R126, R114, R89, L138 et L125, dont les valeurs sont les plus courtes (Fig.2C). L'épi assure une activité photosynthétique importante au cours du remplissage du grain (Berkat, 2005) et sa contribution à la photosynthèse de la plante entière serait comprise entre 13\% et 76\% (Biscoe, 1976).

La longueur du barbe se distingue en quatre groupes : le génotype L150 présente le premier groupe avec la plus longue barbe, suivi par L195. Le groupe intermédiaire englobe les 12 génotypes L172, L125, L 174, R77, R 35, R4, R139, R126, R114, R75, L111 et R89 avec une moyenne variant de $18,7 \mathrm{~cm}$ à 14,30 cm. La plus courte barbe est rassemblé dans le groupe des génotypes L192, L201, L138 et R87 et varie de 12 cm à 14 cm (Fig.2D)

Selon Weyrchi (1995), les barbes sont des feuilles rudimentaires ayant une fonction photosynthétique. De nombreux travaux réalisés sur une large gamme de génotypes semblent confirmer le rôle de la barbe dans le 
remplissage du grain en conditions de déficit hydrique (Gignac, 1965 ; Hadjchristodoulou ,1985; Dib et al., 1990).

L'épi et les barbes sont des facteurs déterminants dans la tolérance au stress hydrique chez le blé par le rôle à la photosynthèse et à la transpiration (Monneveux, 1991). Il s’avère aussi que le rendement est lié positivement à la longueur de l'épi (Kahali, 1995).

Anova à deux facteurs révele des différences trés hautement significatives entre les vingt génotypes (Tableau nº 4 ) .

Tableau $\mathrm{n}^{\circ} 4$ : Analyse de la variance des paramétres morphologiques

\begin{tabular}{|c|c|c|c|c|c|}
\hline Source & ddl & SCM & $\mathrm{CM}$ & F de Fisher & $\operatorname{Pr}>F$ \\
\hline Genotypes & 19 & 3687,174 & 194,062 & 3,788 & $<0,0001^{* * *}$ \\
\hline paramètre & 3 & 518022,490 & 172674,163 & 3370,825 & $<0,0001^{* * *}$ \\
\hline Genot*paramè & 57 & 18244,175 & 320,073 & 6,248 & $<0,0001^{* * *}$ \\
\hline
\end{tabular}

Ces résultats indiquent l'éxistence d’une variabilité assez importante pourront justifier l'analyse génétique pour la sélection. Le test SNK permis de mettre en évidence 7 sous-groupes qui se différencient par leurs aspects morpho-phenologiques: Le premier groupe est formé par le génotype R63 qui se caractérise par une hauteur de la plante et une longueur du col très intéressant. Le $2^{\text {eme }}, 3^{\text {émé }}$ et $4^{\text {éme }}$ groupes forment respectivement les génotypes R34, R77 et L174. Ces génotypes se distinguent par une longueur de l'épi et du col important. Le cinquième groupe constitue de 12 génotypes R4, L111, L195, L125, R89, L138, R126, R114, R87, L139, R75 et R35 moyennement développés. Le sixième groupe comporte les trois génotypes les moins développées L192, L172 et L150. Le dernier groupe (le génotype L201) se distingue par une longueur de l'épi, une hauteur de la plante, une longueur du barbe et une longueur du col moins élevés. Les génotypes appartenant à la variété rechenbachi sont les plus tardifs; caractérisés par la longueur d'épi et du col contrairement aux génotypes de la variété Leucomelan qui sont précoces et plus élevée. Ces derniers se distinguent par la hauteur de la plante et la longueur des barbes. 


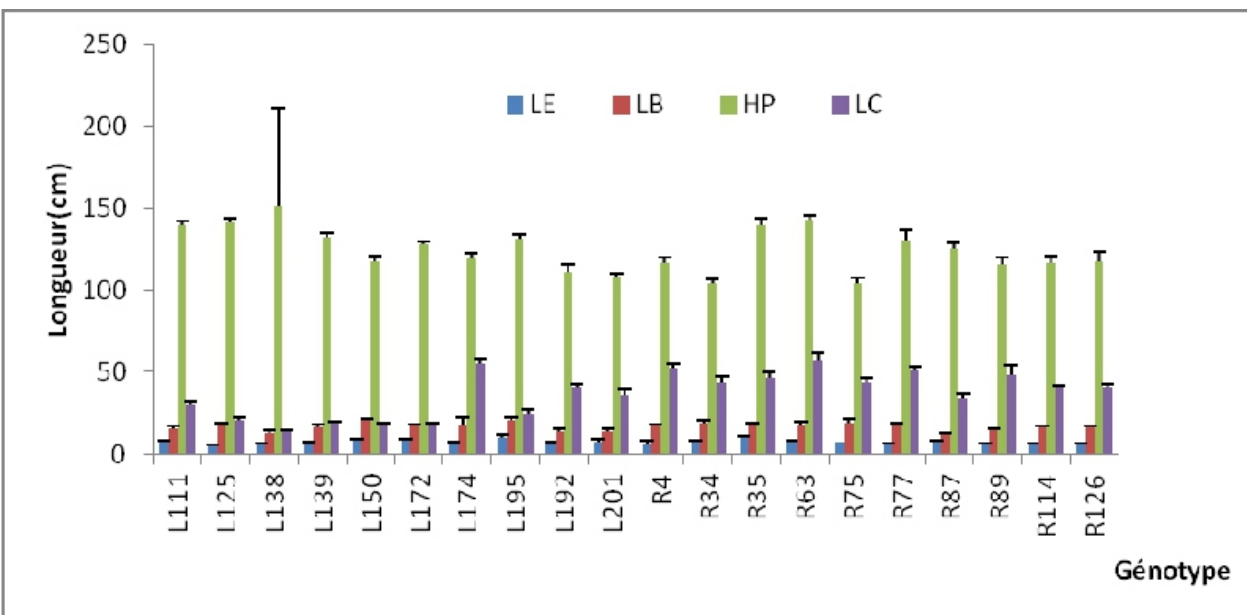

Fig.3. Les paramètres morphologiques récapitulatif chez les vingt génotypes de blé dur.

\section{Analyse des marqueurs moléculaires}

La variabilité génétique à base RAPD nous permet d'analyser les relations entre les génotypes étudiées. Le dépistage initial d'un grand nombre d'amorces RAPD avec de les vingt génotypes blé dur a donné lieu à deux amorces qui peuvent produire des fragments polymorphes informatifs résolubles par électrophorèse sur gel d'agarose (Fig. 4) .

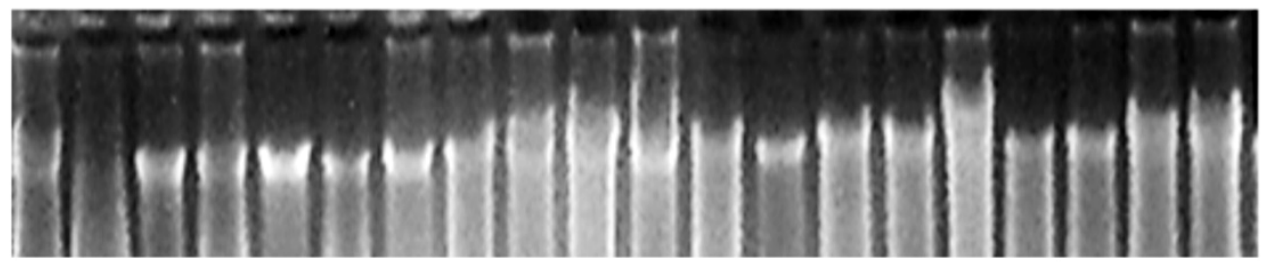

Fig .4. Electrophorèse D’ADN génomique de 20 génotypes de blé dur

L'ADN génomique extrait de génotypes (Fig.4.) est utilisé en tant que modèles pour RAPD-PCR amplification. La selection des amorces est utilisée pour l'identification et la caractérisation génétique des génotypes. Deux amorces ont réussi à donner de bons résultats pour le polymorphisme detecté entre les vingt génotypes. L'amorce DuPw004 révelé deux marqueurs spécifiques pour le génotype L 195 [2459 (+) et 2140 pb (+) pb]. Alors que l'amorce DuPw023 révele quatre marqueurs spécifiques pour le genotype R89 [2558 (+) bp $2459(+)$ bp $2140\left(^{+}\right)$pb, 1700] et un marqueur spécifique pour le genotype R114 (+) pb] (Fig.5,Tableau n 3 ) 

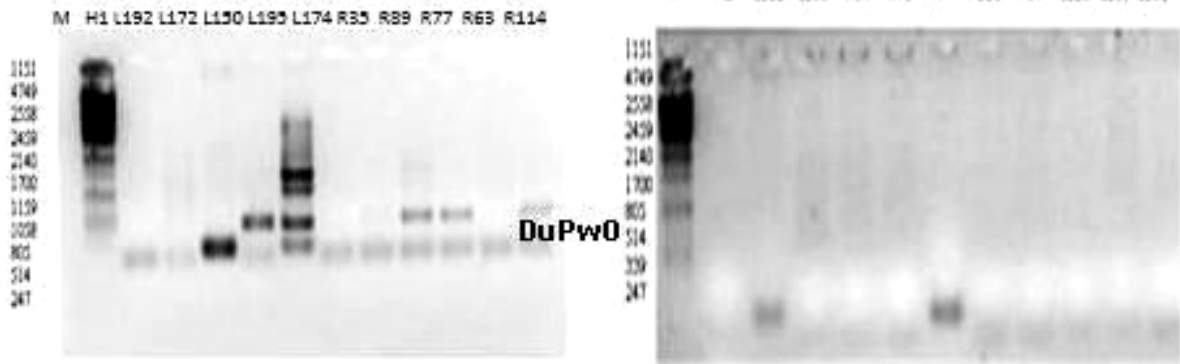

M 18 แม แล

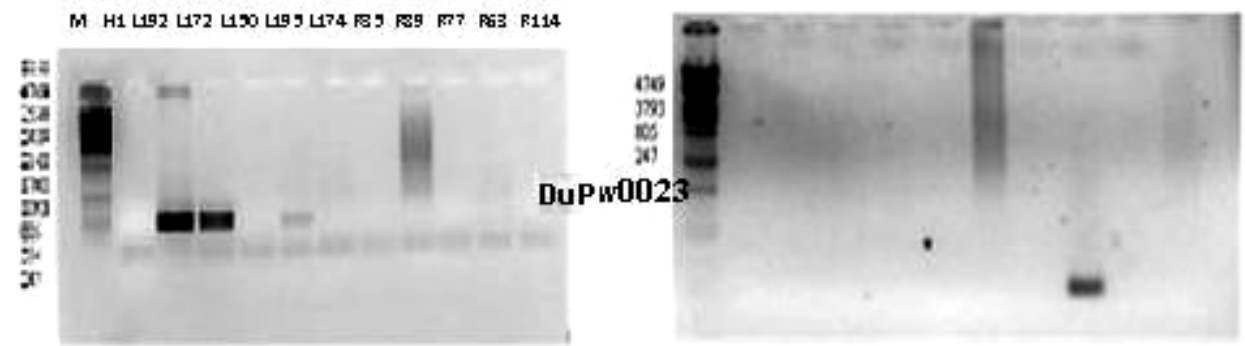

Fig.5. Diagramme éléctrophorétique RAPD des 20 génotypes de blé dur générés par les deux amorces (DuPw004, DuPw023)

Tableau n³ : Analyse RAPD-PCR des 20 génotypes de blé dur générés par les deux amorces DuPw004 et DuPw023

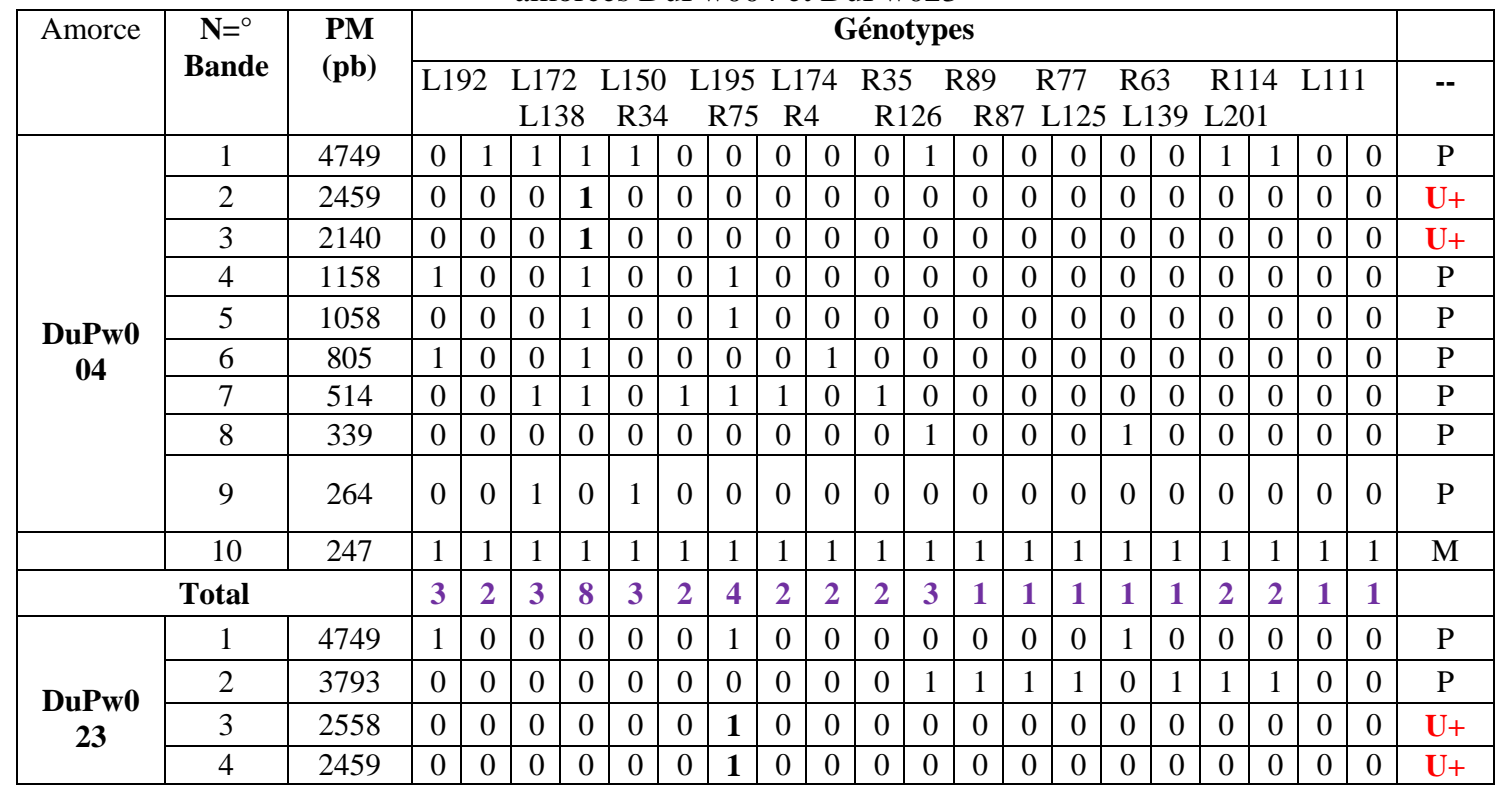




\begin{tabular}{|c|c|c|c|c|c|c|c|c|c|c|c|c|c|c|c|c|c|c|c|c|c|c|c|}
\hline \multirow{6}{*}{} & 2140 & 0 & 0 & 0 & 0 & 0 & 0 & $\mathbf{1}$ & 0 & 0 & 0 & 0 & 0 & 0 & 0 & 0 & 0 & 0 & 0 & 0 & 0 & $\mathrm{U}+$ \\
\hline & 1700 & 0 & 0 & 0 & 0 & 0 & 0 & $\mathbf{1}$ & 0 & 0 & 0 & 0 & 0 & 0 & 0 & 0 & 0 & 0 & 0 & 0 & 0 & $\mathrm{U}+$ \\
\hline & 1093 & 0 & 0 & 0 & 0 & 0 & 0 & 1 & 0 & 1 & 0 & 0 & 0 & 0 & 0 & 0 & 0 & 0 & 0 & 0 & 0 & $\mathrm{P}$ \\
\hline & 8 & 805 & 1 & 1 & 0 & 1 & 0 & 0 & 0 & 0 & 0 & 0 & 0 & 0 & 0 & 0 & 1 & 0 & 0 & 0 & 0 & 0 & $\mathrm{P}$ \\
\hline 9 & 514 & 0 & 0 & 0 & 0 & 0 & 0 & 0 & 0 & 0 & $\mathbf{1}$ & 0 & 0 & 0 & 0 & 0 & 0 & 0 & 0 & 0 & 0 & $\mathrm{U}+$ \\
\hline
\end{tabular}

$\mathrm{P}$ : bande polymorphique, $\mathrm{U}+$ : bande spécifique, $\mathrm{M}$ : bande mono morphique.

L'élément des fragments d'ADN amplifiés se situe entre 0 et 8 bandes. La taille du fragment amplifié varie entre $247 \mathrm{pb}$ et $4749 \mathrm{pb}$. Le nombre des fragments d'ADN obtenu est le même chez les deux amorces. Mais, l'ensemble des bandes est convergente chez tous les génotypes pour les deux amorces. Il varie d'une seule bande chez les deux genotypes L201 et L139 à 10 et 11 bandes chez les deux cultivars L195 et R89 simultanément. Le nombre des bandes amplifiés est exigaux chez plusieurs génotypes intra ou inter varietale. Il varie de 1 à 4 bandes. Seul le genotype L192 presente 6 bandes (Tableau $n^{\circ} 4$ ).

Tableau $\mathrm{n}^{\circ} 4$ : Tableau récapitulatif du nombre des bandes pour chaque génotype avec deux amorces DuPw004 et DuPw023

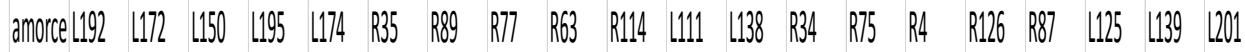

$\begin{array}{llllllllllllllllllllll}\text { DuPNOA4 } & 3 & 2 & 3 & 8 & 3 & 2 & 4 & 2 & 2 & 2 & 3 & 1 & 1 & 1 & 1 & 1 & 2 & 2 & 1 & 1 \\ \text { DuPNOZ3 } & 3 & 2 & 1 & 2 & 1 & 1 & 1 & 1 & 2 & 1 & 1 & 1 & 1 & 1 & 2 & 1 & 2 & 1 & 0 & 0 \\ \text { Total } & 6 & 4 & 4 & 10 & 4 & 3 & 11 & 3 & 4 & 3 & 4 & 2 & 2 & 2 & 3 & 2 & 4 & 3 & 1 & 1\end{array}$

Les 2 amorces ont amplifiées 20 bandes PCR dont 19 bandes sont polymorphes avec un pourcentage de 95\%. L'amorce DuPw004 donne deux bandes uniques et 7 bandes non uniques avec un polymorphisme égal à 88\%. L'amorce DuPw0023 donne 5 bandes uniques et 5 bandes non uniques avec un polymorphisme optimale $100 \%$ (Tableau $\mathrm{n}^{\circ} 5$ ).

Nos résultats concordent avec ceux de Chaib et al. (2015). Ces auteurs ont pu évaluer la variabilité génétique de 10 cultivars de blé dur par analyse de marqueurs RAPD, en utilisant 6 amorces. Ces dernières ont amplifiées 65 bandes PCR dont 43 bandes sont polymorphes avec un pourcentage de $66,15 \%$. L'élément de fragments d'ADN amplifiés se situe entre 0 et 8 . La taille du fragment amplifié a également varié de $222 \mathrm{pb}$ à $2550 \mathrm{pb}$ environ 
Tableau n5: Nombre et le type de l'ADN amplifié et le pourcentage du polymorphisme généré par deux amorces dans une accession de vingt génotypes de blé dur.

\begin{tabular}{|c|c|c|c|c|c|}
\hline \multirow{2}{*}{ Amorces } & \multirow[b]{2}{*}{$\begin{array}{c}\text { Bande } \\
\text { monomorphiques }\end{array}$} & \multicolumn{2}{|c|}{ Bande polymorphique } & \multirow[b]{2}{*}{$\begin{array}{c}\text { Bande } \\
\text { total }\end{array}$} & \multirow{2}{*}{$\begin{array}{c}\% \text { de } \\
\text { polymorphis } \\
\text { me }\end{array}$} \\
\hline & & $\begin{array}{l}\text { Bande } \\
\text { unique }\end{array}$ & $\begin{array}{c}\text { Bande non } \\
\text { unique }\end{array}$ & & \\
\hline DuPw004 & 1 & 2 & 7 & 10 & $88 \%$ \\
\hline DuPw023 & 0 & 5 & 5 & 10 & $100 \%$ \\
\hline
\end{tabular}

La classification de la variété rechenbachi repartit les différents génotypes en cinq principaux cluster: le premier englobe les génotypes R75, R126, R34 et R87, dont les trois génotypes sont identiques avec trés forte similarité 100\%, le genotype R87 est corréle de 66,67\% de similarité avec chaqu'un d'eux. Le deuxième regroupe les trois génotypes R35, R77 et R114 dont les genotypes R35 et R77 présentent une similarité maximale de $100 \%$. Alors que le genotype R114 est corrélé à une similarité équivalente à 66,67\%. Le reste des cultivars R63, R4 et R89; chaque génotype présente un groupe séparé (Tableau n 6, Fig.6).

Sivolap et al., (1997) ont trouvé un pourcentage de polymorphisme de $78 \%$ après une analyse RAPD à 23 variétés de blé dur d'hiver sur un total de 91 produits d'amplification. Le dendrogramme issu de cette analyse révèle des niveaux de similarité distincts entre les différentes variétés.

Tableau $n^{\circ} 6$ : Matrice de la similarité génétique de la variété rechenbachi basée sur la variation PCR-RAPD

\begin{tabular}{|c|c|c|c|c|c|c|c|c|c|c|c|}
\hline & R35 & R89 & & R77 & R63 & R114 & R34 & R75 & R4 & R126 & R87 \\
\hline R35 & 1 & & & & & & & & & & \\
\hline R89 & 0,429 & & 1 & & & & & & & & \\
\hline R77 & 1 & & 0,429 & 1 & & & & & & & \\
\hline R63 & 0,571 & & 0,4 & 0,571 & 1 & & & & & & \\
\hline R114 & 0,667 & & ,286 & 0,667 & 0,286 & 1 & & & & & \\
\hline R34 & 0,4 & & ,154 & 0,4 & 0,333 & 0,4 & 1 & & & & \\
\hline R75 & 0,4 & &, 154 & 0,4 & 0,333 & 0,4 & 1 & 1 & & & \\
\hline R4 & 0,286 & &, 267 & 0,286 & 0,25 & 0,286 & 0,333 & 0,333 & 1 & & \\
\hline R126 & 0,4 & &, 154 & 0,4 & 0,333 & 0,4 & 1 & 1 & 0,333 & 1 & \\
\hline R87 & 0,571 & &, 267 & 0,571 & 0,5 & 0,286 & 0,667 & 0,667 & 0,25 & 0,667 & 1 \\
\hline & & & 0 & 5 & & 10 & 15 & & 20 & 25 & \\
\hline & & Num & +-- & ------+- & & --+-- & -----+- & ------- & --+---- & ------+ & \\
\hline & $\mathrm{K} 7 \mathrm{~S}$ & 7 & & & & & & & & & \\
\hline $\mathrm{Cla}_{\mathrm{a}}$ & $\mathrm{R} 126$ & 9 & & & & & & & & & \\
\hline & F34 & 6 & - & & & & & & & & \\
\hline & Rs? & 10 & & & & & & & & & \\
\hline $\mathrm{Cl}_{23}$ & $\begin{array}{l}\mathrm{R} 35 \\
\mathrm{~K} 7 \mathrm{7}\end{array}$ & 1 & & & & & & & & & \\
\hline & $C_{\mathrm{R} 14}$ & 5 & & & & & & & & & \\
\hline $\mathrm{Cl}_{20}$ & $\prec^{863}$ & 4 & & & & & & & & & \\
\hline $\mathrm{Cla}_{4}$ & $<^{\mathrm{R} 4}$ & 8 & & & & & & & & & \\
\hline $\mathrm{Cla}_{25}$ & ${ }{ }_{\mathrm{K} 99}$ & 2 & & 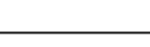 & & & & & & 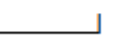 & \\
\hline
\end{tabular}

Fig. 6. Dendrogramme des marqueurs RAPD basé sur la distance Euclidienne des génotypes de la variété rechenbachi. 
La classification hiérarchique des marqueurs moléculaires RAPD répartit les différents génotypes de la variété leucomelan en deux grands cluster distincts de similarité légèrement inférieure à $75 \%$. Le premier grand cluster est divisé en deux sous groupes. Le premier sous groupe englobe les deux génotypes identiques L139 et L201 avec une similarité maximale 100\%. Le deuxième sous-groupe présente les trois génotypes L111, L125 et L138 avec une similarité de $82,9 \%, 76 \%$ et $60 \%$ correlé seccusivement. Le second grand cluster englobe également deux sous groupes; le premier comporte les deux génotypes L150 et L174 avec une trés forte similarité de $86 \%$ lié aux génotypes L172 avec une similarité de $74 \%$ et $60 \%$ respectivement. Le deuxieme sous groupe se distingue par le génotype L192 qui se différe de $80 \%$ et $50 \%$ avec les trois premiers génotypes. Finalement, le génotype L195 en un seul groupe ( $3^{\text {éme }}$ cluster) se differe des autres génotypes de la variété leucomelan avec une similarité qui varie de $0,2 \%$ à $40 \%$ (Tableau n ${ }^{\circ}$ 7, Fig.7).

D’après Abdellatif et Abouzeid.(2011), un polymorphisme de 86,67 $\%$ et $100 \%$ est détecté chez 40 génotypes de blé en utilisant 17 amorces. Le dendrogramme et la Matrice de la similarité génétique basées sur la variation moléculaire montre une forte correlation entre les variétés de blé d'Égypte et celles de la Grèce $\left(r_{1}=0,77\right)$, entre Egyptiens variétés $\left(r_{2}=\right.$ $0,80)$ et entre Grèce variétés $\left(r_{3}=0,74\right)$.

Tableau $n^{\circ} 7$ : Matrice de la similarité génétique de la variété leucomelan basée sur la variation PCR-RAPD

\begin{tabular}{|c|c|c|c|c|c|c|c|c|c|c|}
\hline & 192 L17 & & L150 & L195 & L174 & L111 & L138 & L125 & L139 & L201 \\
\hline 192 & 1 & & & & & & & & & \\
\hline $\mathrm{L} 172$ & 0,52 & 1 & & & & & & & & \\
\hline L150 & 0,236 & 0,6 & 1 & & & & & & & \\
\hline L195 & 0,55 & 0,486 & 0,44 & 1 & & & & & & \\
\hline L174 & 0,28 & 0,7 & 0,867 & 0,314 & 1 & & & & & \\
\hline L111 & $4,00 \mathrm{E}-02$ & 0,4 & 0,333 & 0,143 & 0,4 & 1 & & & & \\
\hline L138 & $1,00 \mathrm{E}-01$ & 0,2 & 0,143 & 0 & 0,2 & 0,6 & 1 & & & \\
\hline L125 & $6,67 \mathrm{E}-02$ & 0,486 & 0,4 & 0,169 & 0,486 & 0,829 & 0,76 & 1 & & \\
\hline L139 & 0,143 & 0,28 & 0,2 & $1,82 \mathrm{E}-02$ & 0,28 & 0,28 & 0,6 & 0,4 & 1 & \\
\hline L201 & 0,143 & 0,28 & 0,2 & $1,82 \mathrm{E}-02$ & 0,28 & 0,28 & 0,6 & 0,4 & 1 & 1 \\
\hline
\end{tabular}

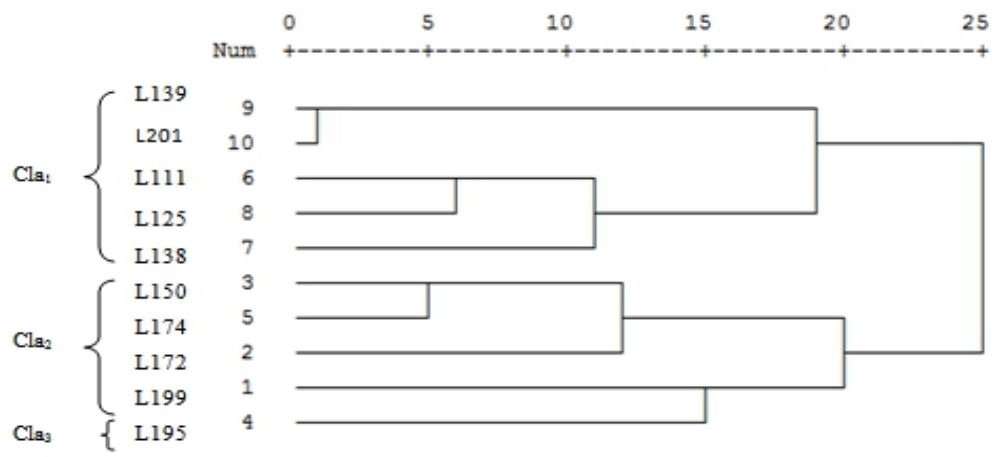

Fig. 7 . Dendrogramme des marqueurs RAPD basé sur la distance Euclidienne des génotypes de la variété Leucomelan. 
La classification hiérarchique globale des deux variétés répartit les différents génotypes en deux grands classes distinctes de similarité équivalente de $76 \%$ à $86 \%$ et séparé par le génotype R4 (3 ${ }^{\text {éme }}$ groupe) qui n’est corrélé à aucun genotype des deux variétes étudiées. La premiere classe comporte 9 genotypes et subdivise en deux sous groupes. Le premier sous groupe représente les deux génotypes identiques L139 et L201 avec trés forte similarité de $100 \%$. Le deuxième est subdivisé en deux sous-sous groupes ou l'un d'entre eux regroupe les quatre génotypes R75, R126, L138 et R34 avec une similarité maximale de $100 \%$ et l'autre comprend les trois génotypes L111, R87 et L125 avec une similarité autour de 50\%. La deuxiéme classe comprend 11 genotypes et subdivise en trois sous groupes. Le premier englobe les trois génotypes L150, L174 et L172 avec une similarité de $100 \%$ et $66,7 \%$ simultanement. Le deuxieme sous groupe englobe les génotypes R35, R77 et R114. Le troixieme regroupe les quatres génotypes L192, R63, L195 et R89 avec une faible correlation entre eux (Tableau nº8, Fig. 8).

Tableau n8 : Matrice de la similarité génétique des deux variétés rechenbachi et leucomelan basées sur la variation PCR-RAPD

\begin{tabular}{|c|c|c|c|c|c|c|c|c|c|c|c|c|c|c|c|c|c|c|c|c|}
\hline & 1.92 & 1772 & L150 & 1995 & 1.774 & B 35 & R8 & Bil & 8663 & Rilly & !illi & 1138 & R34 & $R 79$ & Rit & Ril6 & B897 & 1065 & L.393 & 1201 \\
\hline 19.9 & 1 & & & & & & & & & & & & & & & & & & & \\
\hline 1772 & 0,472 & 1 & & & & & & & & & & & & & & & & & & \\
\hline 1.50 & 0,36 & 0,837 & 1 & & & & & & & & & & & & & & & & & \\
\hline 1998 & 0,471 & 0,535 & 0,447 & 1 & & & & & & & & & & & & & & & & \\
\hline 17.74 & 0,316 & 0,837 & 1 & 0,447 & 1 & & & & & & & & & & & & & & & \\
\hline 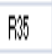 & 0,5 & 0,660 & 0,991 & 0,344 & 0,791 & 1 & & & & & & & & & & & & & & \\
\hline 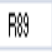 & 0,236 & 0 & 0,149 & 0,033 & 0,49 & 0,344 & 1 & & & & & & & & & & & & & \\
\hline Bi? & 0,632 & 0,478 & 0,8 & 0,44 & 0,6 & 0,791 & 0,49 & 1 & & & & & & & & & & & & \\
\hline B 696 & 0,36 & 0,478 & 0,6 & 0,49 & 0,6 & 0,791 & 0,447 & 0,6 & 1 & & & & & & & & & & & \\
\hline Bill & 0,25 & 0,236 & 0,36 & 0 & 0,36 & 0,480 & 0 & 0,36 & 0,396 & 1 & & & & & & & & & & \\
\hline LiII & .396 & 0,12 & 0.2 & 0.44 & 0,2 & $., 1,158$ & 0,497 & 1,2 & 1,2 & $0,1,158$ & 1 & & & & & & & & & \\
\hline 138 & 0,155 & 0,236 & 0.36 & 0 & 0,36 & 0,488 & 0 & 0,36 & 0,36 & 0,480 & 0,36 & 1 & & & & & & & & \\
\hline B.34 & 0,05 & 0,236 & 0,36 & 0 & 0,36 & 0,480 & 0 & 0,36 & 0,36 & 0,480 & 0,316 & 1 & 1 & & & & & & & \\
\hline 876 & 0,125 & 0,236 & 0,36 & 0 & 0,36 & 0,480 & 0 & 0,36 & 0,36 & 0,480 & 0,36 & 1 & 1 & 1 & & & & & & \\
\hline Rit & 0,36 & 0,12 & 0.2 & 0.48 & 0.2 & 0,158 & 0.148 & 0.2 & 0.2 & $0,1,158$ & 0,2 & 0,158 & $0,1,158$ & $0,1,158$ & 1 & & & & & \\
\hline RiC6 & 0,05 & 0,236 & 0,36 & 0 & 0,36 & 0,480 & 0 & 0.36 & 0,36 & 0,480 & 0,36 & 1 & 1 & 1 & 0,158 & 1 & & & & \\
\hline B887 & 0,189 & 0,679 & 0,837 & 0,269 & 0,837 & 0,661 & 0 & 0,488 & 0,988 & 0,236 & 0,478 & 0,6601 & 0,661 & 0,606 & 0.239 & 0,661 & 1 & & & \\
\hline 1205 & 0 & 0,478 & 0,6 & 0,49 & 0,6 & 0,346 & 0.48 & 0,2 & 0,2 & 0,316 & 0,6 & 0,791 & 0,791 & 0,791 & 0.2 & 0,991 & 0,837 & 1 & & \\
\hline 139 & 0,343 & 0,544 & 0,542 & 0,243 & 0,542 & 0,686 & 0,243 & 0,542 & 0,542 & 0,686 & 0,108 & 0,686 & 0,686 & 0,686 & 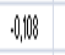 & 0,686 & 0,454 & 0,542 & 1 & \\
\hline 2001 & 0,343 & 0,454 & 0,542 & 0,448 & 0,042 & 0,686 & 0,443 & 0,542 & 0.542 & 0,086 & 0,010 & 0,086 & 0,6866 & 0,686 & 0,0108 & 0,686 & 0,454 & 0,542 & 1 & 1 \\
\hline
\end{tabular}




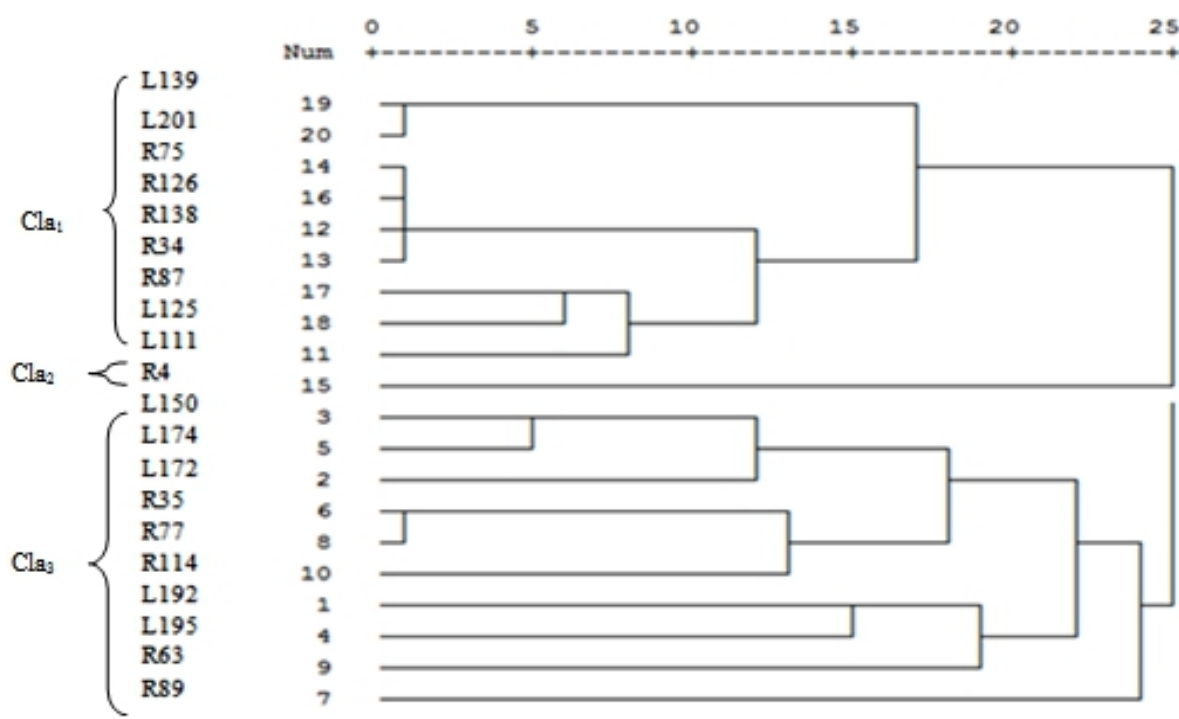

Fig. 8. Dendrogramme des marqueurs RAPD basé sur la distance Euclidienne des 20 génotypes étudiés (rechenbachi et leucomelan).

Notre étude moléculaire des 20 génotypes pour les deux amorces prises ensemble montre un pourcentage élevé de polymorphisme supérieur à $80 \%$, d'ou des génotypes avec une forte similarité (100\%) et autres avec une très faible similarité $(0-0,1 \%)$. Nos résultats coïncident avec les études de Cifci et Yagdi. (2012) sur 16 turquins variétés de blé utilisant des amorces RAPD, la similarité génétique était entre 0,32 et 0,86. Pandey et al. (2015) ont trouvé une similarité génétique de 0.72-0.96 entre des variétés de blé Hexaploïdes.

Naghavi et al. (2004) ont estimé la diversité génétique entre les variétés de blé tendre. Ils ont obtenu un total de 188 bandes par 17 amorces RAPD, et 101 fragments par 35 amorces SSR. Le niveau de polymorphisme était $88 \%$ et $100 \%$ avec RAPD et SSR respectivement. Les marqueurs RAPD utilisés constituent un outil précieux d'efficacité pour l'identification et l'évaluation de la diversité génétique de l'espece et montrent la corrélation entre le polymorphisme et les conditions éco-géographiques (Sharma et al., 2000; Figuliulo, 2000).

Les paramètres étudiés nous ont permis de marquer une grande diversité phénomorphologique et moléculaire entre les génotypes des deux variétés. Ce qui regroupe les génotypes selon leurs différents caractéristiques en quatre groupes: le groupe de deux génotypes précoces L139 et L201 avec une forte similarité et les quatre parametres morphologiques LE, LC, LB et HP moins elevée. Le deuxieme comprend les génotype moyennement developpé R75, R126, R87, L125, L138, L111, L150, R35, L172, R114 et 
R89, caractérisés par HP, LC, LE et LB moyennes. Le troisieme groupe contient les génotypes ayant une LE et LC importants R34, R77 et L174 et une similarité importante de 70\%. Le dernier groupe élucide le génotypes R63 qui se caractérise par une hauteur de la plante et une longueur du col très intéressante et une faible similarité de 0 à 30\% avec les deux génotypes L192 et L195.

\section{Conclusion}

Cette étude, nous a permis de distinguer une variabilité intra et intervariétale. L’identification et le regroupement des deux variétés en classes génotypiques. La classe I se distingue par la présence des génotypes les moins élevés L201. La classe II englobe les génotypes moyennement dévelopés et enfin les génotypes les plus développés R63, R77, L174 et R34.

Nous résultats suggèrent que la diversité génétique dans le blé peut être déterminée par l'utilisation des marqueurs moléculaires. Une variation génétique modérée est observée entre les deux variétés rechenbachi et leucomelan. Cette information pourrait être utile pour les sélectionneurs de blé dur d'utiliser l'échange du matériel génétique des deux variétés, afin d'élargir la variation génétique des accessions de reproduction. En outre, l'utilisation efficace des ressources génétiques dans tous les programmes de sélection végétale nécessite des connaissances plus profondes sur la diversité génétique par d‘autres types de marquage moléculaire.

\section{References:}

Amokrane, A., Bouzerzour ,H., Benmahammed, A.,\& Djekoun, A .(2002) . Caractérisation des variétés locales, Syriennes et européennes de blé dur évaluées Constantine, numéro spécial D , 33 - 38.

Abdellatif,K.F., \& AbouZeid,H.M.(2011). Assessme nt of genetic diversity of Mediterranean bread wheat using Randomly Amplified Polymorphic DNA (RAPD) markers. Journal of Genetic Engineering and Biotechnology (2011) 9, $157-163$.

Barket,H. (2005) . caractérisation morphophysiochimique des descendants issus de cinq générations de Backross et de leurs géniteurs de blé dur (Triticum durum Desf.).Thèse doctorat d’état.ISN.Université Mentouri Constantine.Algerie.

Benbelkacem A et kello.K., 2000. Evaluation du progrès génétique chez quelques variétés du blé dur (Triticum durum. Desf) cultivées en Algérie. Symposium Blé: enjeux \& stratégies : Alger. 7-9 février : 123-132.

Bouzerzour, H., Adjabi, A., Benmahammed, A., Hadj Sahraoui, A.,\& Harkati, N. (2002a). Productivité et adaptation des variétés de céréales en zone semi-aride d’altitude Céréaliculture 37 , 4-12. 
Boudour, L., Djekoun, A., Ghribi, D.,\& Olmi A. (2004). Morphology diversity of Algerian durum wheat population. Arab. Univ. J. sci, 12(2), 597608.

Boudour ,L. (2006). Étude des ressources phyto-génétiques du blé dur (Triticum durumDesf.) algérien : analyse de la diversité génétique et des critères d’adaptation au milieu. Thèse Doctorat d’Etat. Université Mentouri Constantine, 142.

Bouzerzou, H.,\& Oudina.(1989).l'effet des dates et densité des semis sur le rendement de blé et de l’orge dans la region de sétif céréaliculture n¹5,5-19. Biscoe, P.V. (1976). Effet of drought on grain growth. Nature (264) ,541 542.

Chaib , G. Abdelsalem, A.Z.E., Benlaribi, M., Ibrahim M. (2015). Biochemical and molecular Genetic Characterization of ten durum wheat genotypes (Triticum durum Desf)., BIOENG '15 / II. International Bioengineering Conference 25-26 November 2015 Istanbul , Turkey P:163178 - ; ISBN: 978-605-9207-15-7. www.dakam.org.

Cifci, E.A., \& Yagdi, K. (2012). Study of Genetic Diversity in Wheat (Triticum aestivum) Varities Using Random Amplified Polymorphic DNA (Rapd) Analysis. Turk J Field Crops. 17:91-95.

Dekkers, JCM., \&Hospital, F. (2002). The use of molecular genetics in the improvement of agricultural populations [Review]:Natl. Rev. Genet. 3 (1), 22-32.

Dib.A T., (1990). Breeding durum for drough tolerance analytical, synthetical approches and their connections, in wheat breeding prospects and futur approachePanayotov T ; and Pavlov S, (eds). Albena, bulgaria ,224240.

Dellaporta, S.L., Woods, J., \& Hicks, J.B. (1983). A plant DNA minipreparation: Plant Mol. Boil. Rep, 1, 19-21.

Eagles, HA., Bariana ,HS., Ogbonnaya, FC., Rebetzke ,GJ., H o 11 a m b y, GJ., Henry ,RJ., Henschke, PH., \&Carter M . (2001). Implementation of markers in Australian wheat breeding:Aust. J. Agric. Res. 52 (11-12), 13491356.

Figuliulo, G. (2000).A nested analysis to detect relationships between genetic markers and germoplasm classes of durum wheat: Plant Genetic Ressources Neweletter, 124,44- 50.

Grignac, P. (1965). Contribution à l'étude de Triticum durum Desf .Thése de doctorat.Université de toulouse,(pp246).

Hospital, F. (2001). Size of donor chromosome segments around introgressed loci and reduction of linkage drag in marker-assisted backcross programs.Genetics 158(3), p. 1363-1379.

Hadjichristodoulou, A. (1989). Enviromental correlation among grain yield and other important trait of wheat in dry lands.Euphytica, $44,143-150$. 
Kahali, A. (1995).Contribution of carbohydrates and other solutes to osmotic adjustment in wheat leaves under water stress. Plant. Physiol, 145,363-366. Moreau, L., Charcosset, A., Gallais, A. (2001). Efficiency of marker-assisted selection compared with conventional selection. OCL-Ol. Corps Gras Lipides 8 ( 5 ) , p. 496-501.

Monneuveux,p.(1991)-Quelles stratégies pour l’amélioration génétique de la tolérence au déficit hydrique des céréales d’hiver ?In :Chalbi,Demarly Y,eds.L’amélioration des plantespour l'adaptation aux milieux arides.,John libbey Eurotext ,165-86.

Nazco, R., Villegas, D., Ammar, K., Pena, RJ.,Moragues, M.,\&Royo, C. (2012) Can Mediterranean durum wheat landraces contribute to improved grain quality attributes in modern cultivars. Euphytica, 185, pp1-17.

Nachit, M.M., \& Ketata, H. (1991). Selection of morphophysiological traits for multiple abiotic stresses resistance in durum wheat (Triticum turgidum L. Var.durum) in phsiology breeding of winter cereals for stressed mediterranean environments (Montpellier, France 3-6 July 1989) Ed. INRA. Paris (les colloques №55) ,391-400.

Naghavi,M.R.,Mohsen,M.,Ramshini,H.A.,\&Bahman F. (2004). Comparative analyses of the genetic diversity among bread wheat genotypes based on RAPD and SSR markers. Iranian Journal of Biotechnology, 2(3): 195-202. Pandey,A.,Khan,M.K.,Thomas,G.,Hakki,E.E.,Kayis,S.A.,Hamurcu,M.,Gezgi N,S.,Topa,A., \& Akkaya,M.S.(2015). Estimation of Indian and Turkish Hexaploid Wheat Population Structure Employing Molecular Markers. Not Bot Horti Agrobo, 2015, 43(1):70-78.

Sharma, K.D., Singh,B.M.,\& Guleria ,S. (2000).Molecular analysis of variability in Podophyllum-hexan- durum Royle-an endangered medicinal herb of northwestern Himalaya. Plant Genetic-Ressources Newsletter, 124: 57-61.

Sivolap, M., Kutsevich, L.I., Palamarchuk, A.I. \& Totsky, V.N. (1997). Molecular-genetic polymorphism of winter durum wheat determined by polymerase chain reaction with arbitrary primers. Russian-AgriculturalSciences, 1 : 9-13.

Sharma, K.D., Singh,B.M.,\& Guleria ,S. (2000).Molecular analysis of variability in Podophyllum-hexan- durum Royle-an endangered medicinal herb of northwestern Himalaya. Plant Genetic-Ressources Newsletter, 124: 57-61.

Sun, G., Bond,M. H., Nass, R., Martin, Z., Dong.(2003).Theoretical and Applied Genetics 106, 1059-1067.

Weyrchi, R.A. (1995).photosynthesis and water use efficiency of awned and awneletted near isogenic lines of hard winter wheat. Crop Sci. $35,172-176$. 